\title{
Padrões de abundância, riqueza e diversidade de moluscos bivalves na plataforma continental ao largo de Ubatuba, São Paulo, Brasil: uma comparação metodológica
}

\author{
Abílio Soares-Gomes ${ }^{1} \&$ Ana Maria Setubal Pires-Vanin ${ }^{2}$ \\ ${ }^{1}$ Departamento de Biologia Marinha, Universidade Federal Fluminense. Caixa Postal 100.644, 24001-970 Niterói, Rio de \\ Janeiro, Brasil. E-mail: abiliosg@vm.uff.br \\ 2 Instituto Oceanográfico, Universidade de São Paulo. Praça do Oceanográfico 191, 05508-900 São Paulo, São Paulo, Brasil. \\ E-mail: amspires@usp.br
}

\begin{abstract}
Patterns of abundance, richness and diversity of bivalve molluscs from the continental shelf offshore of Ubatuba, São Paulo, Brazil: a methodological comparison. The diversity and richness patterns of bivalve molluscs were studied on the continental shelf of Ubatuba $\left(23^{\circ} 38^{\prime} \mathrm{S}, 45^{\circ} 14^{\prime} \mathrm{W}\right.$ and $\left.23^{\circ} 25^{\prime} \mathrm{S}, 44^{\circ} 51^{\prime} \mathrm{W}\right)$ between 15-120 metres depth. The samples were taken with a rectangular dredge and a beam trawl. The results showed higher values of richness, diversity and evenness for inner shelf (from coast to nearly $50 \mathrm{~m}$ depth) and greater values of abundance for outer shelf (from 50 to around $120 \mathrm{~m}$ depth). This pattern might be due to the higher instability of the inner shelf, which is influenced by the seasonal intrusion of the South Atlantic Central Water (SACW) and the occurrence of cold fronts. Sanders' rarefation curves, distribution and abundance curves and DIMO model were used and the results compared. The Qinghong's model proved to be a good tool for summarizes the results of diversity in a such plural account.
\end{abstract}

KEY WORDS. Biodiversity, community structure, macrobenthos, spatial distribution, soft-bottom.

Uma importante etapa no estudo de comunidades é a busca por padrões de estrutura e composição. O conhecimento de padrões estruturais provê informações que permitem o manejo sustentado de populações e o monitoramento de atividades antrópicas.

Os estudos de fundos não consolidados enfatizam o papel do tipo de sedimento na estruturação das comunidades desses ambientes (e.g. Gray 1974, Alongi \& Christoffersen 1992), sendo granulometria e o teor de matéria orgânica as variáveis sedimentares mais comumente apontadas como as mais importantes (e.g. Pearson \& Rosenberg 1978, Fresi et al._1983, Weston 1988).

Embora seja simplista considerar a granulometria como sinônimo de ambiente físico, devido à composição textural ser resultado de uma complexa interação de fenômenos, tem sido demonstrado que tipos específicos de fundo apresentam correspondência com um grupo de espécies bem definido (Fresi et al. 1983). Não somente a composição qualitativa, mas também parâmetros estruturais das biocenoses, tal como a diversidade específica, parecem estar correlacionados com a estrutura dos sedimentos. Apesar do inquestionável papel dos sedimentos na estruturação do bentos, os efeitos a ele relacionados podem ser mascarados por outras variáveis ambientais, em geral relacionadas à profundidade (WESTON 1988).

A plataforma continental norte do Estado de São Paulo, compreendida entre São Sebastião e Ubatumirim, tem sido alvo de intensivas campanhas oceanográficas desde a década de 1980, visando o conhecimento da estrutura e funcionamento desse ecossistema (Pires-Vanin 1993). Contudo, apesar do considerável volume de informações disponíveis sobre esse ambiente marinho e da importância indiscutível do bentos, as comunidades de bivalves têm sido pouco estudadas, especialmente no aspecto estrutural.

Pode-se examinar a estrutura das comunidades concentrando-se em dois aspectos importantes da sua organização: o número de espécies e as respectivas abundâncias relativas (GILLER 1984). Estas medidas podem ser incorporadas em índices biológicos tais como riqueza, diversidade e uniformidade, na tentativa de resumir as informações e facilitar a comparação intrae entre habitats.

Embora as variações de diversidade das comunidades bênticas possam ser analisadas em diferentes escalas -local, regional e global, as causas dessas variações não estão ainda inteiramente compreendidas, apesar de várias hipóteses terem 
sido formuladas baseadas em fatores diversos, tais como tempo, estabilidade climática, heterogeneidade espacial, distúrbios físicos do ambiente, tipo de sedimento, competição, predação e produtividade. De uma forma geral, em relação a esses fatores, a diversidade tenderia a ser maior em ambientes heterogêneos, sujeitos a uma maior estabilidade climática e a uma freqüência intermediária de ocorrência de distúrbios (Huston 1979, Connel 1978); a maior abundância de predadores diminuiria a competição entre presas, aumentando a diversidade (Wilson 1991, Peterson 1992); ambientes mais produtivos apresentariam maior diversidade, aqual tenderia a aumentar com o passar do tempo (Rex et al. 1993).

A diversidade tem sido medida através de muitos índices, havendo vários métodos para interpretá-la (SANDERS 1968, MAGURRAN 1988). Entretanto, não há uma comparação entre os diversos métodos para verificar se são concordantes na apresentação dos resultados e se estes permitem uma interpretação acurada do ambiente. Além disso, grande parte dos trabalhos existentes referem-se à fauna de região temperada, reconhecida por possuir um menor número de espécies quando comparada à tropical e subtropical.

O presente trabalho visa descrever a estrutura das taxocenoses de bivalves presentes na plataforma continental de Ubatuba, através de seus padrões de abundância, riqueza e diversidade; utilizar diferentes métodos para obtenção desses padrões, verificando sua concordância; definir as medidas de diversidade e riqueza mais adequadas para representar os padrões de distribuição de comunidades bênticas de fundos inconsolidados subtropicais.

\section{MATERIAL E MÉTODOS}

\section{Área de estudo}

A área de estudo situa-se na plataforma continental norte do Estado de São Paulo, entre a Ilha de São Sebastião e a Enseada de Ubatumirim, entre as coordenadas $23^{\circ} 38^{\prime} \mathrm{S}, 45^{\circ} 14^{\prime} \mathrm{W}$ e $23^{\circ} 25^{\prime} \mathrm{S}, 44^{\circ} 51^{\prime} \mathrm{W}$, numa área aproximada de $6.000 \mathrm{Km}^{2}$ (Fig. 1).

Fisiograficamente pode ser dividida em duas porções, a centro-sul e a norte. A porção centro-sul tem como diferencial a presença de obstáculos físicos representados pelas ilhas Anchieta, Vitória, Búzios e São Sebastião, situadas da costa até a isóbata de 50 m (Pires 1992, Pires-V Anin 1993).

Esses anteparos físicos às condições de impacto de mar aberto fazem com que o centro-sul esteja sujeito a um hidrodinamismo menos intenso que a porção norte, o que ocasiona a formação de uma área de deposição de sedimentos finos, especialmente da fração silte-argila, que avança suavemente em direção à plataforma externa. Toda a porção norte e a porção centro-sul externa à isóbata de $50 \mathrm{~m}$ apresentam-se expostas diretamente à influência do mar aberto e, portanto, sujeitas a um hidrodinamismo mais acentuado, que se reflete no padrão deposicional preponderante dessa área, as areias (Fig. 2).

A característica oceanográfica principal da plataforma sudeste e sul brasileira, e que estrutura os ecossistemas de fundo (CApítoli 1998, Pires-Vanin 2001), é a presença sazonal da Água Central do Atlântico Sul (ACAS) sobre a plataforma interna, área que vai da costa até cerca de $50 \mathrm{~m}$ de profundidade. Durante o verão, a ACAS, que flui para o norte sob a Corrente do Brasil, penetra na plataforma junto ao fundo, alcançando a região costeira; durante o inverno, ela se retrai em direção à

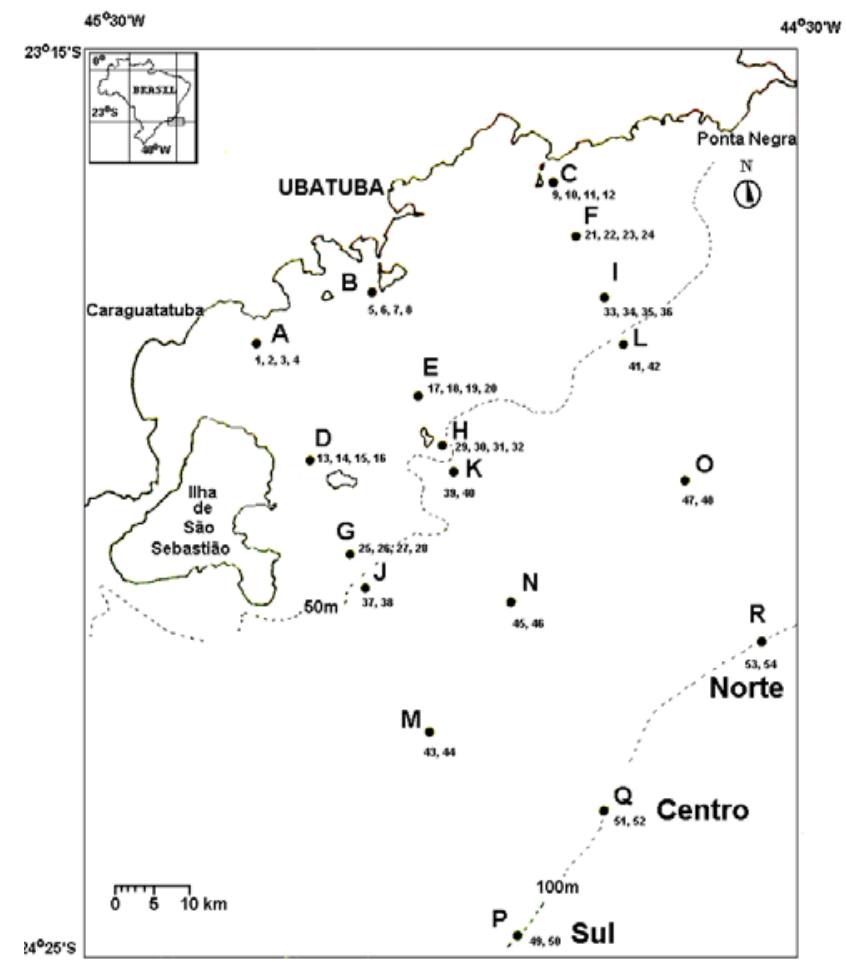

Figura 1. Área de estudo com localização das estações de coleta.

margem continental, sendo substituída na plataforma interna pela Água Costeira (AC), quente e menos salina. No verão instala-se uma termoclina marcante à meia-água devido a presença de água fria junto ao fundo e água aquecida no restante da coluna (CASTRO-Filho et al. 1987). Com a retração da ACAS durante o inverno a distribuição da temperatura na plataforma interna torna-se homogênea, variando entre 20 e $25^{\circ} \mathrm{C}$ (PIRESVANIN \& MatSUURA 1993).

\section{Metodologia}

As amostras foram obtidas em 18 estações situadas ao longo de três radiais perpendiculares à costa, situadas em profundidades entre 15 e $120 \mathrm{~m}$, em dezembro de 1986 e julho de 1987, a bordo das embarcações Veliger II e N/Oc. "prof. W. Besnard", ambos da Universidade de São Paulo (Fig. 1). As amostras foram agrupadas por faixas batimétricas definidas conforme se segue: faixa I, de 15 a $25 \mathrm{~m}$; faixa II, de 30 a $38 \mathrm{~m}$; faixa III, de 40 a $48 \mathrm{~m}$; faixa IV, $50 \mathrm{~m}$; faixa V, de 70 a $79 \mathrm{~m}$ e faixa VI, de 100 a $120 \mathrm{~m}$. As estações situadas entre 15 e $48 \mathrm{~m}$ foram consideradas pertencentes à plataforma interna e as restantes, à plataforma externa. Para a coleta de sedimentos utilizou-se um pegador de fundo vanVeen de $0,1 \mathrm{~m}^{2}$ e para as amostras biológicas empregou-se uma draga de arrasto retangular e uma rede tipo "beam trawl", ambas com malhagem de $11 \mathrm{~mm}$ no ensacador interno e de $20 \mathrm{~mm}$ no ensacador externo.

Cerca de $200 \mathrm{~g}$ do sedimento coletado pelo pegador de fundo foram separados para análise granulométrica e de matéria orgânica dissolvida, realizadas de acordo com metodologia 

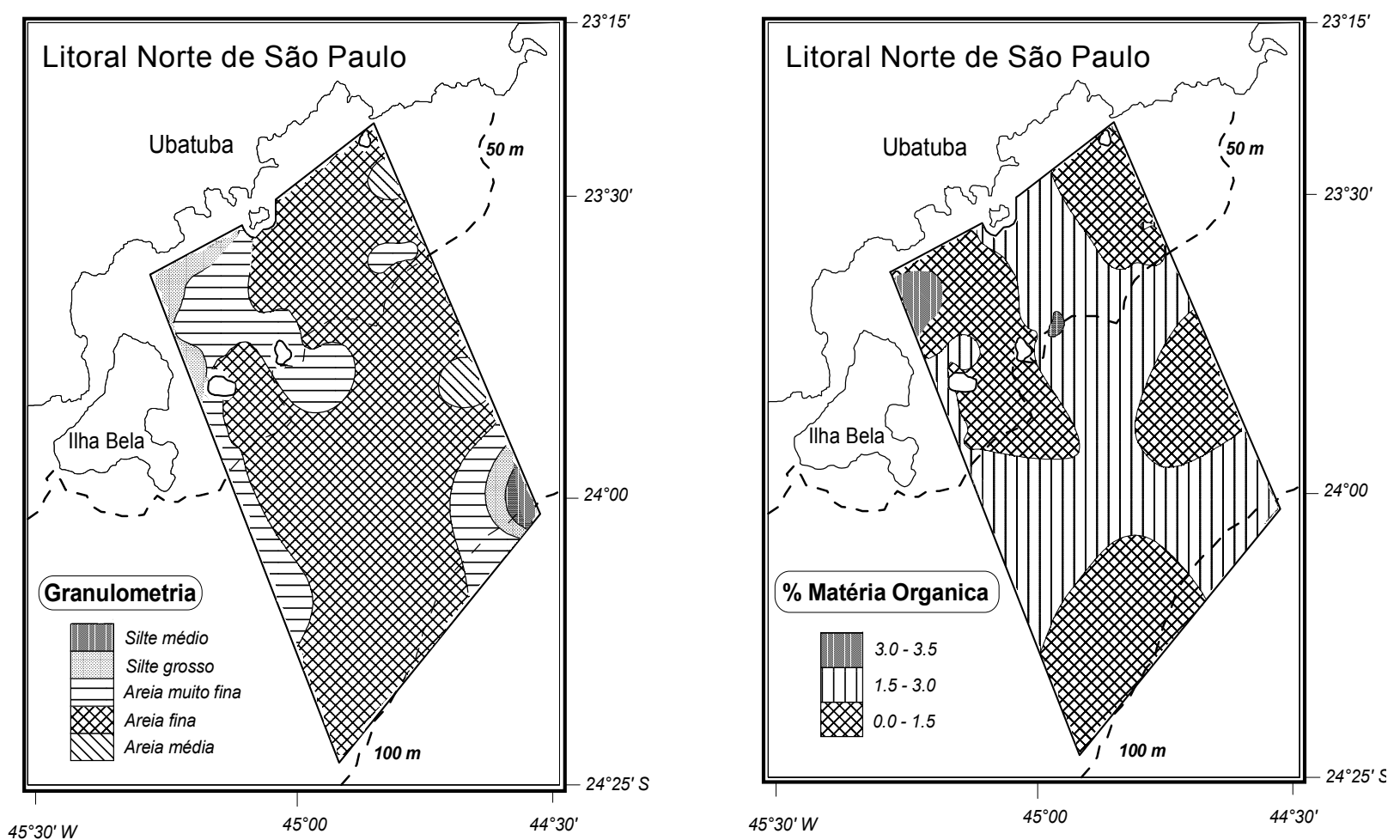

Figura 2. Distribuição do diâmetro médio dos grãos e matéria orgânica dos sedimentos na área de estudo. Ilha Bela = Ilha de São Sebastião.

padrão de peneiramento e pipetagem descrita em SugUIO (1973). De cada arrasto foram amostrados 20 litros de sedimento para posterior lavagem através de uma série de peneiras graduadas (malhas de 2,0,1,0 e 0,5 $\mathrm{mm}$ ) e retirada dos bivalves.

Neste trabalho utilizou-se uma abordagem pluralista, procurando-se comparar o resultados obtidos por várias técnicas com aqueles provenientes dos índices de Shannon e Pielou, por estes serem os mais usados em ecologia bêntica. Os métodos utilizados foram: curva de rarefação de Sanders, curvas de distribuição e abundância e modelo DIMO. A convergência de resultados de várias técnicas serve como teste de robustez, permitindo um grande grau de certeza sobre a estrutura espacial ou temporal da comunidade.

As curvas de rarefação de Sanders foram calculadas pelo método de Hulbert (1971); as curvas de distribuição de abundância foram construídas conforme GILLER (1984); a diversidade e a equitatividade foram obtidas através dos índices de diversidade de Shannon e uniformidade de Pielou, respectivamente. Os valores de diversidade foram submetidos ao método de Hutcheson (1970) para teste de sua significância estatística. Visando resumir as informações, os valores de Shannon $\left(\mathrm{H}^{\prime}\right)$ foram plotados contra $\mathrm{o} \log _{2}$ da riqueza (S), para a construção de um modelo gráfico tipo DIMO (Diversity Model) (QINGHONG, 1995), obtendo-se num mesmo plano gráfico informações relativas à diversidade, riqueza e uniformidade.

O diâmetro médio dos grãos e a concentração de matéria orgânica foram representados por mapas de distribuição de isolinhas, construídos com o auxílio do aplicativo para microcomputadores "Surfer for Windows 6.01", utilizando-se o método de interpolação de Krigen.

\section{RESULTADOS}

O número mínimo, médio e máximo de indivíduos e de espécies coletados nas diferentes faixas batimétricas são mostrados nas figuras 3 e 4 , respectivamente. Os maiores valores médios e as maiores amplitudes de variação do número de indivíduos ocorreram nas faixas batimétricas IV e VI pertencentes à plataforma externa. O número total e o número médio de espécies e a amplitude de variação dessa variável foram muito semelhantes nas diferentes faixas batimétricas amostradas.

A tabela I apresenta os valores de dominância das espécies nas diferentes faixas batimétricas. Três espécies apresentaram dominância expressiva, variando entre aproximadamente 40 e $60 \%$, sendo cada uma delas dominante em uma determinada faixa de profundidade da plataforma externa: Corbula cubaniana ORbIGNY, 1842 na faixa IV, Corbula patagonica OrbIGNY, 1846 na faixa V e Cyclinella tenuis (RéCluz, 1852) na faixa VI. Nas demais faixas batimétricas as espécies apresentaram baixa dominância, inferior a $22 \%$, estando distribuídas em quase todo o gradiente batimétrico da plataforma interna. As espécies principais foram: Abra lioica (DALL, 1881) na faixa I, Ctena orbiculata (MonTAGU, 1808) nas faixas I e II, Corbula caribaea ORBIGNy, 1842 nas faixas I e III e Periploma ovata Orbigny, 1842 nas faixas II e III. 

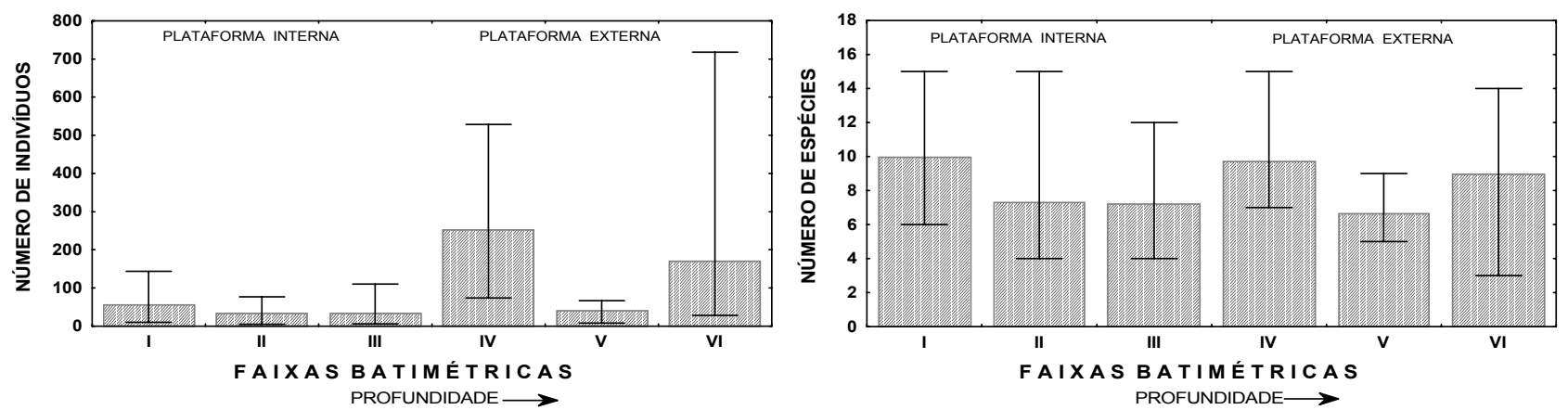

Figuras 3-4. Valores médios do número de indivíduos (3) e de espécies (4) nas diferentes faixas batimétricas e sua amplitude de variação indicada pela barra de erros. Faixas I a III, plataforma interna; faixas IV a VI, plataforma externa.

Tabela I. Dominância numérica (\%) das espécies de bivalves nas faixas batimétricas da região. As espécies com maiores valores de dominância, em cada faixa, estão destacadas em negrito.

\begin{tabular}{|c|c|c|c|c|c|c|}
\hline \multirow{2}{*}{ Espécies } & \multicolumn{6}{|c|}{ Faixas batimétricas } \\
\hline & 1 & II & III & IV & V & $\mathrm{VI}$ \\
\hline Abra lioca (Dall, 1881) & 14,82 & 4,57 & 1,72 & 0,00 & 0,00 & $<1,00$ \\
\hline Adrana electa (A.Adams, 1846) & 1,05 & 0,00 & 0,00 & 0,00 & 0,00 & 0,00 \\
\hline Adrana patagonica (Orbigny, 1846) & 1,65 & 5,08 & 3,44 & 9,19 & 0,00 & 0,00 \\
\hline Anadara brasiliana (Lamarck, 1819) & 9,28 & 1,01 & $<1,00$ & $<1,00$ & 0,00 & 0,00 \\
\hline Anadara notabilis (Röding, 1798) & 1,00 & 0,00 & 0,00 & 0,00 & 0,00 & 0,00 \\
\hline Anadara ovalis (Brugière, 1789) & 2,84 & 0,00 & 0,00 & 0,00 & 0,00 & 0,00 \\
\hline Americuna besnardi Klappenbach, 1962 & 0,00 & 9,39 & 0,00 & $<1,00$ & 0,00 & 6,61 \\
\hline Amiantis purpuratus (Lamarck, 1818) & 1,00 & 0,00 & 0,00 & 0,00 & 0,00 & 0,00 \\
\hline Atrina seminuda (Orbigny, 1846) & 1,00 & 0,00 & 0,00 & 0,00 & 0,00 & 0,00 \\
\hline Cardiomya perrostrata (Dall, 1881) & 0,00 & $<1,00$ & $<1,00$ & 3,04 & 0,00 & $<1,00$ \\
\hline Carditamera floridana Conrad, 1838 & 0,00 & 0,00 & $<1,00$ & 0,00 & 2,55 & 0,00 \\
\hline Carditamera micella Penna, 1971 & 0,00 & $<1,00$ & 1,47 & 5,62 & 12,34 & 7,98 \\
\hline Chione cancelata (Linnaeus, 1767) & 1,00 & 0,00 & 2,46 & 0,00 & 0,00 & 0,00 \\
\hline Chlamys tehuelchus (Orbigny, 1846) & 4,34 & 2,79 & 0,00 & $<1,00$ & 1,00 & 1,07 \\
\hline Corbula caribaea Orbigny, 1842 & 13,77 & 6,34 & 15,23 & 2,18 & 0,00 & 0,00 \\
\hline Corbula cubaniana Orbigny, 1853 & 0,00 & 1,52 & 1,00 & 39,65 & 0,00 & $<1,00$ \\
\hline Corbula dietziana C.B. Adams, 1852 & 1,00 & $<1,00$ & $<1,00$ & 0,00 & 0,00 & 0,00 \\
\hline Corbula lyoni Pilsbry, 1897 & 0,00 & 0,00 & 7,62 & $<1,00$ & 1,00 & 0,00 \\
\hline Corbula patagonica Orbigny, 1846 & 1,50 & 3,30 & 1,96 & 15,86 & 56,17 & 3,70 \\
\hline Cosa brasiliensis Klappenbach, 1966 & 0,00 & 0,00 & 9,09 & 0,00 & 3,83 & $<1,00$ \\
\hline Crassatella riograndensis Vokes, 1973 & 0,00 & 0,00 & 0,00 & 1,65 & 0,00 & 4,38 \\
\hline Crassinela marplatensis Castellanos, 1970 & 1,00 & 2,54 & $<1,00$ & 0,00 & 2,55 & 0,00 \\
\hline Crassinela martinicensis (Orbigny, 1842) & 4,49 & 4,31 & $<1,00$ & $<1,00$ & 1,28 & 0,00 \\
\hline Crenella divaricata (Orbigny, 1946) & 0,00 & 1,01 & $<1,00$ & 0,00 & 1,00 & $<1,00$ \\
\hline Ctena orbiculata (Montagu, 1808) & 12,27 & 13,20 & $<1,00$ & 0,00 & 0,00 & 0,00 \\
\hline
\end{tabular}

Revista Brasileira de Zoologia 20 (4): 717-725, dezembro 2003 
Tabela I. Continuação.

\begin{tabular}{|c|c|c|c|c|c|c|}
\hline \multirow{2}{*}{ Espécies } & \multicolumn{6}{|c|}{ Faixas batimétricas } \\
\hline & 1 & II & III & IV & $\mathrm{V}$ & $\mathrm{VI}$ \\
\hline Cuspidaria platensis E. A. Smith, 1915 & 0,00 & 0,00 & 0,00 & $<1,00$ & 0,00 & $<1,00$ \\
\hline Cyclinella tenuis (Récluz, 1852) & 0,00 & 0,00 & 0,00 & 0,00 & $<1,00$ & 58,56 \\
\hline Entodesma alvarezi Orbigny, 1846 & 1,00 & $<1,00$ & $<1,00$ & $<1,00$ & 1,28 & $<1,00$ \\
\hline Ervilia concentrica (Holmes, 1860) & 1,00 & 0,00 & 0,00 & 0,00 & 0,00 & 0,00 \\
\hline Felaniella candeana (Orbigny, 1842) & $<1,00$ & 0,00 & 0,00 & 0,00 & 0,00 & 0,00 \\
\hline Limaria thryptica (Penna, 1971) & $<1,00$ & 0,00 & $<1,00$ & 0,00 & 0,00 & 0,00 \\
\hline Limatula hendersoni Olsson \& McGinty, 1958 & 0,00 & 0,00 & $<1,00$ & 0,00 & 0,00 & $<1,00$ \\
\hline Limopsis antillensis Dall, 1881 & 0,00 & 0,00 & 0,00 & 0,00 & 1,00 & 2,33 \\
\hline Linga amiantus (Dall, 1901) & 1,00 & 0,00 & 0,00 & 0,00 & 0,00 & 0,00 \\
\hline Macoma tenta (Say, 1834) & 5,69 & 2,03 & 4,42 & $<1,00$ & 0,00 & 0,00 \\
\hline Mactra janeiroensis E. A .Smith, 1915 & 2,24 & $<1,00$ & 5,90 & 0,00 & 0,00 & 0,00 \\
\hline Mactrelona alata (Spengler, 1802) & 2,84 & $<1,00$ & 0,00 & 0,00 & 0,00 & 0,00 \\
\hline Musculus lateralis (Say, 1822) & 0,00 & $<1,00$ & 0,00 & $<1,00$ & 4,25 & $<1,00$ \\
\hline Nucula puelcha Orbigny, 1846 & 4,19 & 6,34 & $<1,00$ & 2,18 & 3,40 & $<1,00$ \\
\hline Nucula semiornata Orbigny, 1846 & 1,00 & 0,00 & 1,72 & 0,00 & 0,00 & 0,00 \\
\hline Nuculana larranagai Klappenbach \& Scarabino, 1968 & 0,00 & 0,00 & 0,00 & 0,00 & 4,68 & 9,14 \\
\hline Nuculana platessa (Dall, 1889) & 0,00 & 0,00 & 0,00 & 0,00 & 0,00 & 1,17 \\
\hline Ostrea puelchana Orbigny, 1841 & 0,00 & 0,00 & 0,00 & $<1,00$ & 0,00 & 0,00 \\
\hline Pandora bushiana Dall, 1886 & 0,00 & $<1,00$ & $<1,00$ & 15,53 & 1,00 & 1,26 \\
\hline Periploma compressa Orbigny, 1846 & 3,29 & 3,30 & 1,47 & $<1,00$ & 0,00 & 0,00 \\
\hline Periploma ovata Orbigny, 1846 & 7,03 & 13,20 & 21,13 & $<1,00$ & 1,28 & $<1,00$ \\
\hline Pitar rostratus (Kock, 1844) & 2,24 & 8,12 & 11,06 & $<1,00$ & 0,00 & 0,00 \\
\hline Pleuromeris sanmartini Klappenbach, 1971 & 0,00 & 0,00 & 2,21 & 0,00 & 0,00 & $<1,00$ \\
\hline Poromya cymata Dall, 1889 & 0,00 & 0,00 & $<1,00$ & 1,25 & 0,00 & $<1,00$ \\
\hline Pteria hirundo (Linnaeus, 1758) & 0,00 & 0,00 & 0,00 & $<1,00$ & 1,28 & $<1,00$ \\
\hline Raeta plicatela (Lamarck, 1818) & 1,00 & 0,00 & 0,00 & 0,00 & 0,00 & 0,00 \\
\hline Semele casali Doello-Jurado, 1949 & 0,00 & $<1,00$ & 0,00 & 0,00 & 0,00 & 0,00 \\
\hline Semele proficua (Pulteney, 1799) & 1,00 & 0,00 & 0,00 & 0,00 & 0,00 & 0,00 \\
\hline Solemya patagonica E. A. Smith, 1885 & 0,00 & 1,52 & 1,23 & 0,00 & 0,00 & 0,00 \\
\hline Tellina martinicensis Orbigny, 1853 & 1,20 & 0,00 & 0,00 & 0,00 & 0,00 & 0,00 \\
\hline Tellina versicolor De Kay, 1843 & 1,00 & 5,84 & $<1,00$ & 0,00 & 1,00 & 0,00 \\
\hline Timothynus rehderi (Altena, 1968) & 0,00 & 0,00 & 0,00 & 0,00 & 0,00 & $<1,00$ \\
\hline Trachycardium muricatum (Linnaeus, 1758) & 1,00 & $<1,00$ & $<1,00$ & $<1,00$ & 0,00 & $<1,00$ \\
\hline Verticordia ornata (Orbigny, 1842) & 0,00 & 0,00 & 0,00 & 0,00 & 1,00 & $<1,00$ \\
\hline
\end{tabular}

Na plataforma interna as curvas de distribuição de abundância das espécies foram pouco inclinadas em relação ao eixo $y$, o que revela uma distribuição relativamente uniforme nas faixas I, II e III (Fig. 5). Na plataforma externa, faixas IV, V e VI, houve uma dominância mais marcante, caracterizada por curvas com maior inclinação em relação ao eixo y (Fig. 6).

As curvas de rarefação obtidas mostraram que os maiores valores de riqueza ocorreram na plataforma interna, com o 

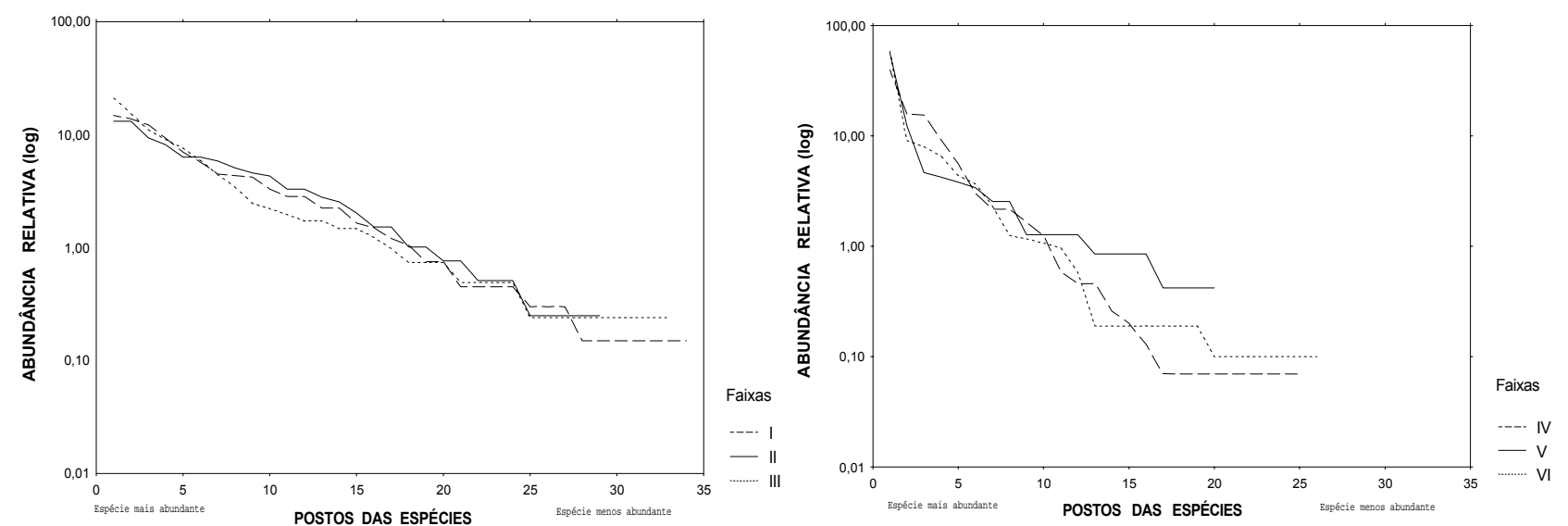

Figuras 5-6. Curvas de distribuição de abundância de indivíduos por espécies, em ordem decrescente de dominância, nas faixas batimétricas da plataforma interna.

número esperado de espécies variando entre 20 e 34. Para a plataforma externa esse número situou-se entre 10 e 12 (Fig. 7).

A tabela II apresenta os valores de diversidade e uniformidade para cada faixa batimétrica considerada. As maiores diversidades e uniformidades foram obtidas na plataforma interna e nesta área, com exceção da faixa III, todas as demais faixas apresentaram uma diferença altamente significativa em relação às da plataforma externa, conforme indicado pelos valores obtidos no teste " $\mathrm{t}$ " (Tab. III). A diversidade não apresentou diferenças significativas entre as três faixas da plataforma interna e entre as três faixas da plataforma externa.

De um modo geral, considerando-se todos os métodos utilizados, a plataforma interna apresentou maiores valores de diversidade, riqueza e uniformidade quando comparada com a plataforma externa (Fig. 8).

Tabela II. Valores de diversidade de Shannon e uniformidade de Pielou, por faixa batimétrica.

\begin{tabular}{lcccccc}
\hline & Faixa I & Faixa II & Faixa III & Faixa IV & Faixa V & Faixa VI \\
\hline Shannon & 2,80 & 2,80 & 2,66 & 1,90 & 1,76 & 1,62 \\
Pielou & 0,79 & 0,85 & 0,76 & 0,59 & 0,59 & 0,49 \\
\hline
\end{tabular}

Tabela III. Valores de "t" de Student calculados pelo método de Hutcheson (1970).

\begin{tabular}{cllllll}
\hline & $\mathrm{I}$ & $\mathrm{II}$ & $\mathrm{III}$ & $\mathrm{IV}$ & $\mathrm{V}$ & $\mathrm{VI}$ \\
\hline $\mathrm{I}$ & 1 & 0,33 & 0,71 & $4,00^{*}$ & $5,04^{*}$ & $5,12^{*}$ \\
II & & 1 & 1,12 & $4,58^{*}$ & $5,80^{*}$ & $5,76^{*}$ \\
III & & & 1 & 3,37 & $4,33^{*}$ & $4,49^{*}$ \\
IV & & & & 1 & 0,56 & 1,05 \\
V & & & & & 1 & 0,58 \\
\hline VI & & & & & & 1 \\
\hline
\end{tabular}

* Valores altamente significativos.

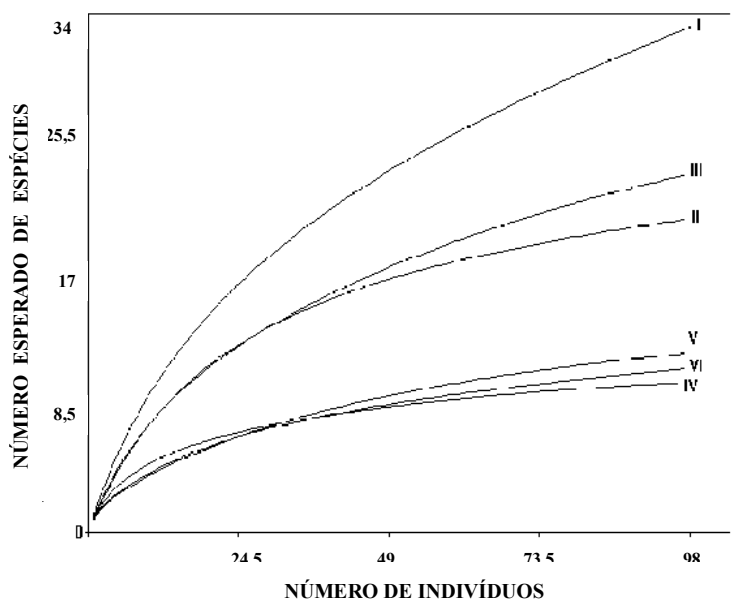

Figura 7. Curvas de rarefação de Sanders representando a riqueza de espécies nas diferentes faixas batimétricas.

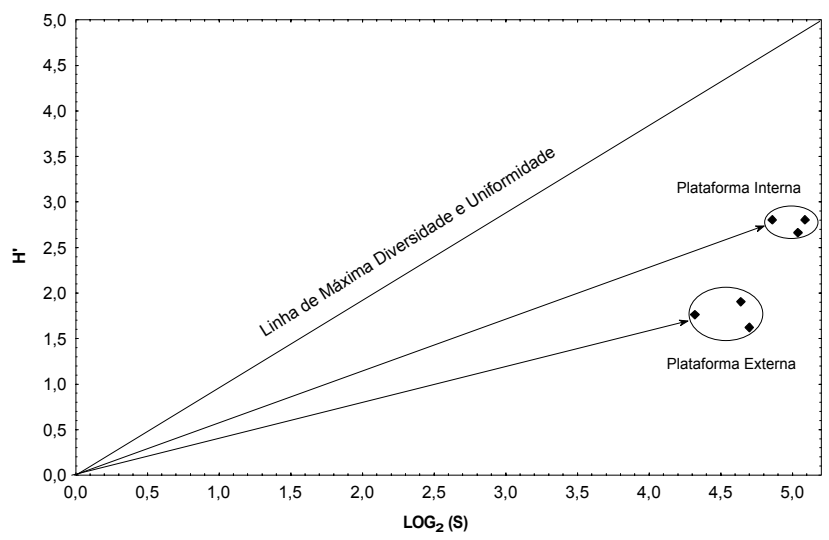

Figura 8. Modelo de diversidade de QINGHONG (1995) aplicado às taxocenoses de moluscos bivalves da região de Ubatuba.

Revista Brasileira de Zoologia 20 (4): 717-725, dezembro 2003 


\section{DISCUSSÃO}

O presente estudo mostrou que a fauna de bivalves pode ser empregada com segurança na representação da estrutura de comunidades do bentos em geral, tal como acontece com Polychaeta, outro grupo da macrofauna freqüentemente utilizado para tal (Alongi \& Christoffersen 1992, Paiva 1993). Os bivalves apresentaram uma maior diversidade na plataforma interna, até cerca de $50 \mathrm{~m}$ de profundidade, enquanto que as maiores densidades ocorreram a partir da isóbata de $50 \mathrm{~m}$. À semelhança do obtido por PIres-VANin (1993) para a macrofauna total da plataforma continental ao largo de Ubatuba e São Sebastião, respectivamente, os maiores valores de diversidade não se relacionaram necessariamente a um tipo de sedimento.

Distúrbios do sedimento decorrentes de tempestades e ação de ondas podem ter uma grande influência na composição de espécies das comunidades bênticas. Mudanças na composição da comunidade resultam tanto de efeitos diretos sobre a sobrevivência das espécies quanto de efeitos indiretos, tais como mudanças nas características sedimentares e modificação das atividades de bioturbação. Entretanto, os efeitos sobre o bentos podem variar em função da duração do distúrbio, da freqüência e duração de eventos relativos ao recrutamento e da história de vida dos organismos (Posey et al. 1996).

Segundo Pires-VANIN (1993), a estrutura do macrobentos da plataforma ao largo de Ubatuba pode ser relacionada aos padrões hidrodinâmicos atuantes nessa área da plataforma, que é regido por fenômenos sazonais relacionados a massas de água e penetração de frentes frias. A plataforma interna é uma região mais instável devido à intrusão da ACAS no verão e sua retração no inverno, o que modifica periodicamente a salinidade, a temperatura e o teor de oxigênio dissolvido. Durante o outono e o inverno a área está submetida a freqüentes passagens de frentes frias, o que ocasiona revolvimento do fundo por ação de correntes de marés e ondas de maior amplitude. Já a plataforma externa encontra-se sob a influência constante da ACAS, o que confere uma maior estabilidade à coluna de água, ao substrato e, conseqüentemente, ao bentos.

Os maiores valores de riqueza e diversidade relatados por Pires-VANin (1993) para a macrofauna total e os encontrados neste trabalho para os bivalves, podem ser relacionados à menor estabilidade existente na plataforma interna. Segundo Grassle (1989), distúrbios locais, de pequena escala, podem manter uma maior diversidade devido à formação de agregados de espécies, que se desenvolvem em ciclos de não-equilíbrio em diferentes escalas de tempo. Na plataforma interna de Ubatuba, possíveis fontes desses distúrbios têm sido relatadas: entrada periódica da ACAS, desalojando ou causando a mortalidade de espécies estenotérmicas (SANTos \& Pires-VANIN 1999); entrada maciça na área do siri Portunus spinicarpus (Stimpson, 1871), espécie agressiva e carnívora que pode aumentar a mortalidade do bentos pelo aumento de predação (Pires 1992); aumento do hidrodinamismo local causado pela entrada de frentes frias, capaz de provocar a morte de algumas espécies através de remoção do seu habitat natural ou por soterramento e asfixia.

A variação na diversidade dos invertebrados bênticos tem sido frequentemente associada ao gradiente de profundidade e às características sedimentares. O trabalho de ABSALÃo (1991), realizado com moluscos da plataforma sul brasileira, mostrou que no gradiente batimétrico estudado pelo autor, entre 11 e $50 \mathrm{~m}$, valores mais elevados foram encontrados na porção externa e em areias finas heterogêneas. A diversidade aumentou da costa em direção ao mar aberto. Nesse trabalho, altos valores de diversidade estiveram ligados à maior riqueza de bivalves e não ao componente uniformidade. Contudo, condições externas à plataforma podem alterar essa relação. Sumida \& Pires (1997), estudando a diversidade na região do talude, área mais profunda e adjacente à da plataforma de Ubatuba aqui investigada, mostraram haver uma redução da diversidade da fauna de moluscos, com a ocorrência de apenas um terço do número de espécies relatadas para a plataforma externa.

Outros trabalhos com moluscos na costa sul brasileira (ABSALÃo 1987, GonÇALVES \& LANA 1991) não apresentam dados relativos à diversidade, o que impossibilita comparações com os nossos resultados. Além disso, um confronto entre amostras provenientes de diferentes regiões geográficas deve ser feito com cautela, especialmente quando se verificam diferenças em relação ao esforço amostral e ao emprego de instrumentos de coleta distintos. Em decorrência dessas diferenças, é possível fazer apenas algumas comparações sobre o número de espécies que ocorrem em áreas distintas, num mesmo tipo de habitat.

A grande maioria dos trabalhos ecológicos realizados com bivalves na costa sudeste/sul apresentou uma riqueza entre 45 e 60 espécies, contrastando com um mínimo de 13 espécies para a região de Rio Grande, Rio Grande do Sul (ABSALÃo 1987) e um máximo de 118 para a região de Campos, Rio de Janeiro (C. Miyagi, com. pess.). Essas duas localidades estão situadas nos extremos geográficos da costa sudeste/sul, mantendo entre si uma distância de aproximadamente $13^{\circ}$ de latitude. Embora seja esta uma primeira avaliação sobre a variação geográfica da diversidade de taxocenoses de bivalves nessa área, o padrão obtido é o mesmo do encontrado por LAMbShead (1993) e Vincent \& Clark (1995) para os bivalves e a macrofauna total do Atlântico Norte, ou seja, observa-se um aumento taxon-específico da diversidade em direção a baixas latitudes.

Estudos sobre diversidade mantêm-se em evidência nas diversas áreas da ecologia, havendo um incremento de trabalhos devotados a esse assunto nas últimas décadas; apesar disso, observa-se um considerável desacordo sobre a metodologia mais adequada para mensuração da diversidade, que permita obtenção e interpretação mais precisas dos resultados.

Washington (1984), May (1975) e Pеet (1974), entre outros, discutem os vários índices e técnicas disponíveis para a medida de diversidade. Baev \& Penev (1993) e Magurran (1988) apresentam mais de 20 métodos adotados para diversidade alfa e uniformidade. Essa profusão está relacionada ao fato de que a maioria deles apresenta restrições ou falhas ao medir amostras com características diferentes em relação à abundância, dominância, riqueza específica e número total de indivíduos. Em face da grande variedade de técnicas disponíveis, faz-se necessário uma orientação objetiva sobre a escolha da mais adequada.

Não existe medida universal de diversidade, segundo Baev \& Penev (1993). Esses autores advogam uma abordagem pluralista para o estudo de tal fator, a qual irá permitir que sejam revelados os múltiplos aspectos da variação das associações biológicas no espaço e no tempo. Igualmente favorável 
a esse argumento, ToKeshi (1993) afirma que a descrição e a interpretação dos padrões de abundância de espécies são incapazes de fornecer, sozinhos, uma compreensão não ambígua das comunidades, e que abordagens analíticas não podem alcançar esse objetivo separadamente.

Os resultados do presente trabalho mostraram uma grande convergência nos resultados das várias técnicas utilizadas. Tal fato permitiu resumir as informações no modelo de QINGHONG (1995) que utiliza os índices de Shannon e Pielou. Caso se confirme o bom desempenho destes índices em outros estudos de abordagem pluralista sobre a estrutura do bentos marinho de fundos inconsolidados, o modelo de Qinghong poderá vir a ser adotado como padrão, permitindo comparações objetivas e eficientes entre diferentes comunidades.

\section{REFERÊNCIAS BIBLIOGRÁFICAS}

AbSALÃo, R.S. 1987. Associações malacológicas ao largo do Rio Grande (RS). As comunidades paralelas de Thorson e associações bênticas de Pérès. Anais do Simpósio sobre Ecossistemas da Costa Sul e Sudeste Brasileira, Cananéia, p. 401-414.

ABSALÃo, R.S. 1991. Environmental discrimination among softbottom mollusc associations off Lagoa dos Patos, South Brazil. Estuarine, Coastal and Shelf Science, London, 32: 71-85.

Alongi, D.M. \& P. Christoffersen. 1992. Benthic infauna and organism-sediment relations in a shallow tropical area: influence of outwelled mangrove detritus and physical disturbance. Marine Ecolology Progress Series, Amelinghausen, 81: 229-245.

Baev, A. \& J. Penev. 1993. BIODIV. Program for calculating biological diversity parameters, similarity, niche overlap, and cluster analysis. Version 4.1. Pensoft, Sofia.

Capítoli, R. 1998. Bentos da Plataforma Continental, p. 131134. In: U. Seeliger; C. Odebrecht \& J.P. Castello (Eds). Os ecossistemas costeiro e marinho do extremo sul do Brasil. Rio Grande, Ecoscientia, XII+326p.

Castro-Filho, B.M.; L.B. Miranda \& S.Y. Myao. 1987. Condições hidrográficas na plataforma continental ao largo de Ubatuba: variações sazonais e em média escala. Boletim do Instituto Oceanográfico, São Paulo, 35 (2): 135-151.

ConnelL, J.H. 1978. Diversity in tropical rainforest and coral reefs. Science, Washington, D.C., 199: 1302-1310.

Fresi, E.; M.C. Gambi; S. Focardi; R. Bargagli; F. Baldi \& L. Falsiai. 1983. Benthic community and sediment types: a structural analysis. P.S.Z.N.I.: Marine Ecology, Nápoles, 4 (2): 101121.

GiLLER, P.S. 1984. Community Structure and Niche. London, Chapman \& Hall, 176p.

GonÇalves, E.M. \& P.C. Lana. 1991. Padrões de distribuição de bivalvia e gastropoda na plataforma continental da costa sudeste do Brasil $\left(24^{\circ} \mathrm{S}-27^{\circ} \mathrm{S}\right)$. Nerítica, Curitiba, 6 (1-2): 73-92.

Grassle, J.F. 1989. Species diversity in deep-sea communities. Trends in Ecology and Evolution, Amsterdam, 4: 12-15.

GrAY, J.S. 1974. Animal-sediment relationship. Oceanography and Marine Biology Annual Review, Aberdeen, 12: 223261.
HULbert, S.H. 1971. The nonconcept of species diversity: a critique and alternative parameters. Ecology, Durham, 52: 577586.

Huston, M. 1979. A general hypothesis of species diversity. The American Naturalist, Chicago, 113: 81-101.

HutCHESON, K. 1970. A test for comparing diversities based on Shannon's formula. Journal of Theoretical Biology, London, 29: 151-154.

LAMBSHEAD, P.J.D. 1993. Recent developments in marine biodiversity research. Oceánis, Paris, 19 (6): 5-24.

Magurran, A.E. 1988. Ecological diversity and its measurement. New Jersey, Princeton University Press, X+179p.

MAY, R.M. 1975. Patterns of Species Abundance and Diversity, p. 81-120. In: M.L Cody \& J.M. Diamond (Eds). Ecology and Evolution of Communities. Cambridge, Belknapp Press, 543p.

PaIva, P.C. 1993. Anelídeos poliquetos da plataforma continental norte do Estado de São Paulo: I. Padrões de densidade e diversidade específica. Boletim do Instituto Oceanográfico, São Paulo, 41(1/2): 69-80.

Pearson, T.H. \& R. Rosenberg. 1978. Macrobenthic succession in relation to organic enrichment and pollution of the marine environment. Oceanography and Marine Biology Annual Review, Abedeen, 16: 229-311.

PeEt, R.K. 1974. The measurement of species diversity. The American Naturalist, Chicago, 100: 65-75.

Peterson, C.H., 1992. Competition for food and its communitylevel implications. Benthos Research, Hokkaido, 42: 1-11.

PIRES, A.M.S. 1992. Structure and dynamic of benthic megafauna on the continental shelf offshore of Ubatuba, Southeastern Brazil. Marine Ecology Progress Series, Amelinghausen, 86: 63-76.

Pires-VAnin, A.M.S. 1993. A macrofauna bêntica da plataforma continental ao largo de Ubatuba, São Paulo, Brasil. Publicação especial do Instituto Oceanográfico, São Paulo, 10: 137-158.

. 2001. Isopod assemblages on the continental shelf and upper slope from the southwestern Atlantic, p. 289300. In: B. Kensley \& R.C. Brusca (Eds). Crustacean Issues 13. Isopod Systematics and Evolution. Rotterdan, A.A.Balkema, VIII+357p.

Pires-Vanin, A.M.S. \& Y. Matsuura. 1993. Estrutura e função do ecossistema de plataforma continental da região de Ubatuba, Estado de São Paulo: uma introdução. Publicação especial do Instituto Oceanográfico, São Paulo, 10: 1-8.

Posey, M.; W. Lindberg; T. Alphin \& F. Vose. 1996. Influence of storm disturbance on an offshore benthic community. Bulletin of Marine Science, Miami, 59 (3): 523-529.

QINGHONG, L. 1995. A model for species diversity monitoring at community level and its application. Environmental Monitoring and Assessment, Dordrecht, 34: 271-287.

Rex, M.A.C.T.; R.R.Hessler; J.A. Allen; H.L.SAnders \& G.D.F WiLson. 1993. Global-scale latitudinal patterns of species diversity in the deep-sea benthos. Nature, London, 365: 639-649.

SANDERS, H.L. 1968. Marine benthic diversity: a comparative study. American Naturalist, Chigaco, 102 (925): 243-282.

SAntos, M.F.L. \& A M.S.Pires-Vanin. 1999. The Cumacea com- 
munity of the southeastern Brazilian continental shelf: structure and dynamics. Scientia Marina, Barcelona, 63 (1): $15-25$.

Suguio, K.1973. Introdução à sedimentologia. São Paulo, Edgard Blücher/EDUSP, 317p.

Sumida, P.Y.G. \& A.M.S. Pires-Vanin. 1997. Benthic Associations of the shelfbrake and upper slope off Ubatuba-SP, southeastern Brazil. Estuarine, Coastal and Shelf Science, London, 44: 779-784.

ТокеSHI, M. 1993. Species abundance patterns and community structure. Advances in Ecological Research, London, 24: 111-186.
VinCENT, A. \& A. ClaRke. 1995. Diversity in the marine environment. Trends in Ecology and Evolution, Amsterdam, 10 (2): $55-56$

WASHINGTON, H.G. 1984. Diversity, biotic, and similarity indices: a review with special relevance to aquatic ecosystems. Water Research, Amsterdam, 18 (6): 635-694.

Weston, D.P. 1988. Macrobenthos-sediment relationships on the continental shelf off Cape Hatteras, North Carolina. Continental Shelf Research, Oxford, 8 (3): 267-286.

WiLson, W.H. 1991. Competition and predation in marine softsediment communities. Annual Review of Ecology and Systematics, Palo Alto, 21: 221-241.

Recebido em 21.III.2003; aceito em 17.XI.2003. 